\title{
Determination of group velocity of propagation of Lamb waves in aluminium plate using piezoelectric transducers
}

\author{
Z. Lašováa ${ }^{a, *}$, R. Zemčík ${ }^{a}$

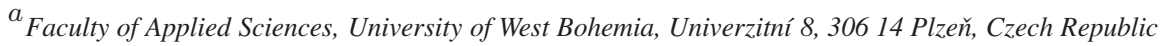

Received 2 March 2017; accepted 2 June 2017

\begin{abstract}
A prior knowledge of group velocities of Lamb wave modes is a key for analysis of time signals in guidedwave based structural health monitoring. The identification of multiple wave modes may be complicated due to dependency of group velocity on frequency (dispersion). These dependencies for infinite plate of constant thickness can be calculated by a numerical solution of analytic equation. Two alternative approaches to determine group velocities of zero-order Lamb wave modes in aluminum plate were used in this work: Two-dimensional Fast Fourier Transform (2D-FFT) and methods of time-frequency processing. 2D-FFT requires a high number of time signals in equidistant points, therefore it was applied on data from finite element analysis of wave propagation in the plate. Group velocities for chosen frequencies were also determined using wavelet transform (WT) of signals as differencies of times of arrival measured by a pair of piezoelectric transducers. The results from 2D-FFT and wavelet transform were compared to the analytic solution.
\end{abstract}

(C) 2017 University of West Bohemia. All rights reserved.

Keywords: Lamb waves, piezoelectric transducers, 2D-FFT, wavelet transform

\section{Introduction}

Lamb waves attracted scientific interest in recent years due to their potential use in the structural health monitoring (SHM, automated structural defect detection). They are a type of elastic waves propagating in plates or hollow cylinders, so Lamb waves-based SHM can be successfully used for shell structural parts (such as aircraft fuselage [3], pressure vessels, wind turbine blades etc.), or for monitoring of pipelines.

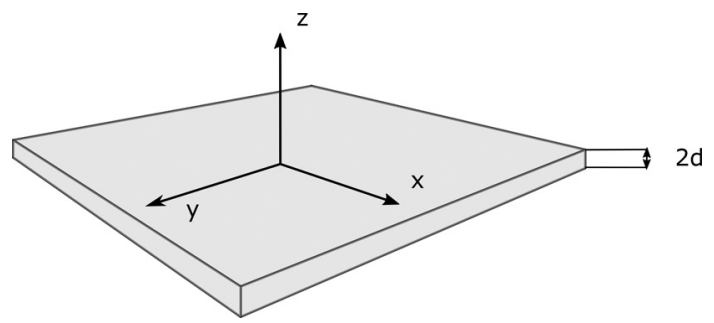

Fig. 1. Coordinate system of the plate

Considering Lamb waves in plates, plane of particle motion is defined by the direction of propagation and the normal to the plate (e.g. plane $x-z$ in Fig. 1 with $x$ as the direction of propagation). In contrast to bulk waves existing in two wave modes (longitudinal, transverse

*Corresponding author. Tel.: +420 377632 327, e-mail: zlasova@kme.zcu.cz. https://doi.org/10.24132/acm.2017.346 
shear), Lamb waves have generally infinite number of modes. These modes are divided to two groups: symmetric $\left(S_{i}\right)$ and antisymmetric $\left(A_{i}\right)$, with respect to the middle plane. It is advantageous to use low frequency range, where only two zero-order modes exist (denoted as $S_{0}$ and $A_{0}$ ). With increasing frequency higher modes occur. The determination of group velocities of the Lamb modes is the key for identification of waves reflected by a damage [13] or to locate the sources of acoustic emission [12].

Lamb waves are dispersive, which means their group and phase velocities depend on frequency. These dependencies for each Lamb wave mode are called dispersion curves and can be calculated by numerical solution of Rayleigh-Lamb equation [5]

$$
\frac{\tan \beta d}{\tan \alpha d}=-\left[\frac{4 \alpha \beta k^{2}}{\left(k^{2}-\beta^{2}\right)}\right]^{ \pm 1},
$$

where the exponent +1 refers to the symmetric modes and -1 to the antisymmetric modes. The coefficients $\alpha, \beta$ are defined by

$$
\alpha=\sqrt{\frac{\omega^{2}}{c_{L}^{2}}-k^{2}} \text { and } \beta=\sqrt{\frac{\omega^{2}}{c_{T}^{2}}-k^{2}} .
$$

In equations (1) and (2), $\omega$ is angular frequency, $k$ is wavenumber, $d$ is half-thickness of the plate, $c_{L}$ is velocity of longitudinal wave and $c_{T}$ is velocity of transverse shear wave.

Rayleigh-Lamb equation applies on theoretical model of infinite isotropic plate of constant thickness. An alternative method of approximation of dispersion curves applicable on any isotropic thin-walled structure was developed: two-dimensional Fast Fourier Transform (2DFFT) presented by Alleyne and Cawley [1]. The input data for the 2D-FFT are time-records in multiple positions along the direction of propagation of the wave. Input data can be collected by measurement (by moving laser $[4,10]$ or air-coupled ultrasonic probe [6]) or by numerical solution $[2,8]$. In this work, finite element model of the cross-section of the plate was used to collect data for 2D-FFT.

The dispersion curves for group velocities can be also determined for given frequencies by direct measurement of time of arrival of single frequency components. In this case it is advantageous to excite waves, in which this frequency component dominates. This can be achieved by choice of proper actuating signal. From the signals collected by sensors, the significant frequency component is separated by means of signal processing methods. Wavelet Transform (WT) is preferred by most of authors [9,13], Wigner-Ville Distribution [14] or ShortTime Fourier Transform [15] were also proposed. In this case wavelet transform was chosen, because it provides best determination of time-of-arrival of acoustic event among mentioned methods. This is thanks to variable size of window function (wavelet) and similarity of wavelet function with actuated sine burst.

In this paper, dispersion curves were calculated by 2D-FFT of numerical results. Group velocities for a set of input frequencies in range $100-500 \mathrm{kHz}$ were determined by timefrequency analysis of signals from piezoelectric transducers. Calculated and measured group velocities were compared with the analytical dispersion curves.

\section{Determination of dispersion curves by 2D-FFT}

Due to the fact that Lamb waves are periodical both in temporal and spatial domain, two dimensional Fast Fourier Transform (2D-FFT) can be used for transformation of time-spatial 
data to wavenumber-frequency domain. If $x$ is the direction of propagation of Lamb waves, 2D-FFT is defined as

$$
H(k, f)=\int_{-\infty}^{\infty} \int_{-\infty}^{\infty} u(x, t) \mathrm{e}^{-\mathrm{i}(k x+t)} \mathrm{d} x \mathrm{~d} t
$$

where $f$ is frequency. The matrix $u(x, t)$ is composed of time responses (displacements $u_{x}, u_{z}$ ) in equidistant points along $x$. To achieve sufficient spatial resolution, a high number of these "sensors" must be used.

Finite element model of the aluminum plate was created to collect the data for 2D-FFT. The model was a cross-section $x, z$ of the plate and plane strain was assumed, which corresponds to the character of Lamb waves. The thickness $2 d=2 \mathrm{~mm}$ corresponded to an experimental plate used later and the finite elements were four-node quadrilaterals of size $0.25 \mathrm{~mm}$. Material parameters of the plate are presented in Table 1. Static material parameters were used - tensile modulus and Poisson's ratio measured by a tensile test of set of 3 specimens cut from the plate.

Table 1. Material parametrs of the aluminum plate

\begin{tabular}{llll}
\hline Tensile modulus & $E$ & {$[\mathrm{GPa}]$} & 69.2 \\
Poisson's ratio & $\nu$ & {$[-]$} & 0.287 \\
Density & $\rho$ & {$\left[\mathrm{kg} \cdot \mathrm{m}^{-3}\right]$} & 2600 \\
\hline Longitudinal wave velocity & $c_{L}$ & {$\left[\mathrm{~m} \cdot \mathrm{s}^{-1}\right]$} & 5884 \\
Shear wave velocity & $c_{S}$ & {$\left[\mathrm{~m} \cdot \mathrm{s}^{-1}\right]$} & 3216 \\
\hline
\end{tabular}

Abaqus Explicit v.6-14 was used for the simulation of wave propagation. The time increment was set to $\Delta t=4.0 \times 10^{-8} \mathrm{~s}$. The model was loaded at one end by a time-dependent displacement (Hann) pulse with duration $2.0 \times 10^{-6} \mathrm{~s}$ (presented in Fig. 2). Its frequency spectrum was sufficient to excite broadband frequency response.
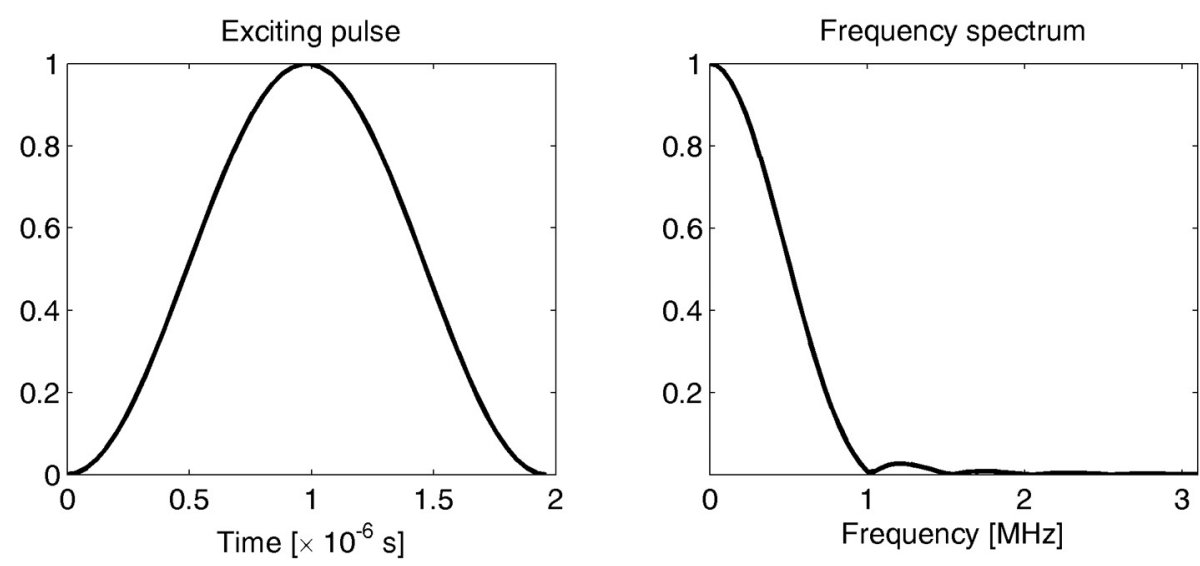

Fig. 2. Exciting pulse and its frequency spectrum (normalised amplitudes)

It turned out to be advantageous to excite the zero-order symmetric and antisymmetric modes separately. To excite unique mode, displacement-based load across the thickness was used. The magnitudes of the displacement were determined by solution of Rayleigh-Lamb equation. Only the dominant displacement directions were used as boundary conditions for each mode:

$$
u_{z}^{0}(z)=A k \frac{\sin \beta z}{\cos \beta h}+2 \frac{\alpha \beta \sin \alpha z}{\left(k^{2}-\beta^{2}\right) \cos \alpha h}
$$


in the case of symmetric mode $S_{0}$ and

$$
u_{x}^{0}(z)=A i \beta \frac{\sin \beta z}{\sin \beta h}-2 k^{2} \frac{\sin \alpha z}{\left(k^{2}-\beta^{2}\right) \sin \alpha h}
$$

for antisymmetric mode $A_{0}$.

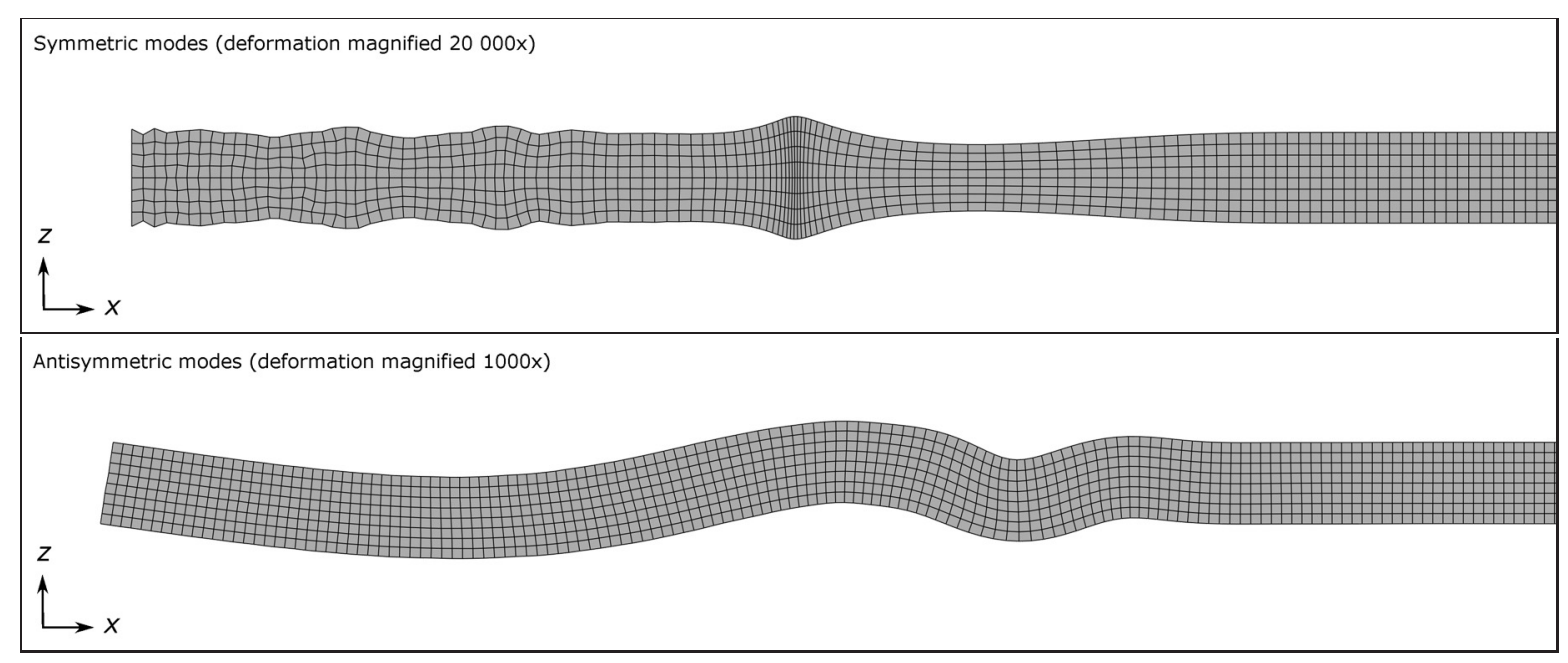

Fig. 3. Deformation of cross-section of the plate for case of symmetric and antisymmetric modes

The deformation of cross-section of the plate for both cases is presented in Fig. 3. Time histories of displacements at 4096 nodes on the top surface of the plate were ordered as columns of the matrix $u(x, t)$. Longitudinal displacement $u_{x}$ was used for determination of symmetric modes and transversal displacement $u_{z}$ for antisymmetric modes. In both cases, the matrix $u(x, t)$ was transformed by 2D-FFT to wavenumber-frequency domain and the resulting matrices $H(k, f)$ are visualised as colour plots in Fig. 4a-b. Elements with higher intensities (darker shade of grey) indicate admissible combinations of frequency and wavenumber, i.e. they approximately show the dispersion curves in $k-f$ domain. In given frequency range $0-1 \mathrm{MHz}$, zero-order symmetric mode $S_{0}$ and antisymmetric modes $A_{0}$ and $A_{1}$ are clearly visible. For comparison, theoretical dispersion curves (calculated by DCTool, a program for numerical solution of Rayleigh-Lamb equation [7]) are superimposed to the 2D-FFT results, as is shown in Fig. 4c-d. It is apparent that numerical results correspond very well with the analytic solution.

2D-FFT was also used for determination of group velocities. The group velocity generally defined by

$$
c_{g(n)}=\frac{\partial \omega}{\partial k}
$$

was calculated as a ratio of differences

$$
c_{g(n)}=\frac{\omega_{n+1}-\omega_{n-1}}{k_{n+1}-k_{n-1}} .
$$

The values of group velocities were determined for selected frequencies in range $100-500 \mathrm{kHz}$ (to ensure the presence of zero-order modes only) with step of $10 \mathrm{kHz}$. The wavenumbers were found from the maximum values in rows of matrix $H(k, f)$ corresponding to closest values of the given frequencies. The calculated group velocities are presented along with experimental data in Fig. 9 in the next section. 

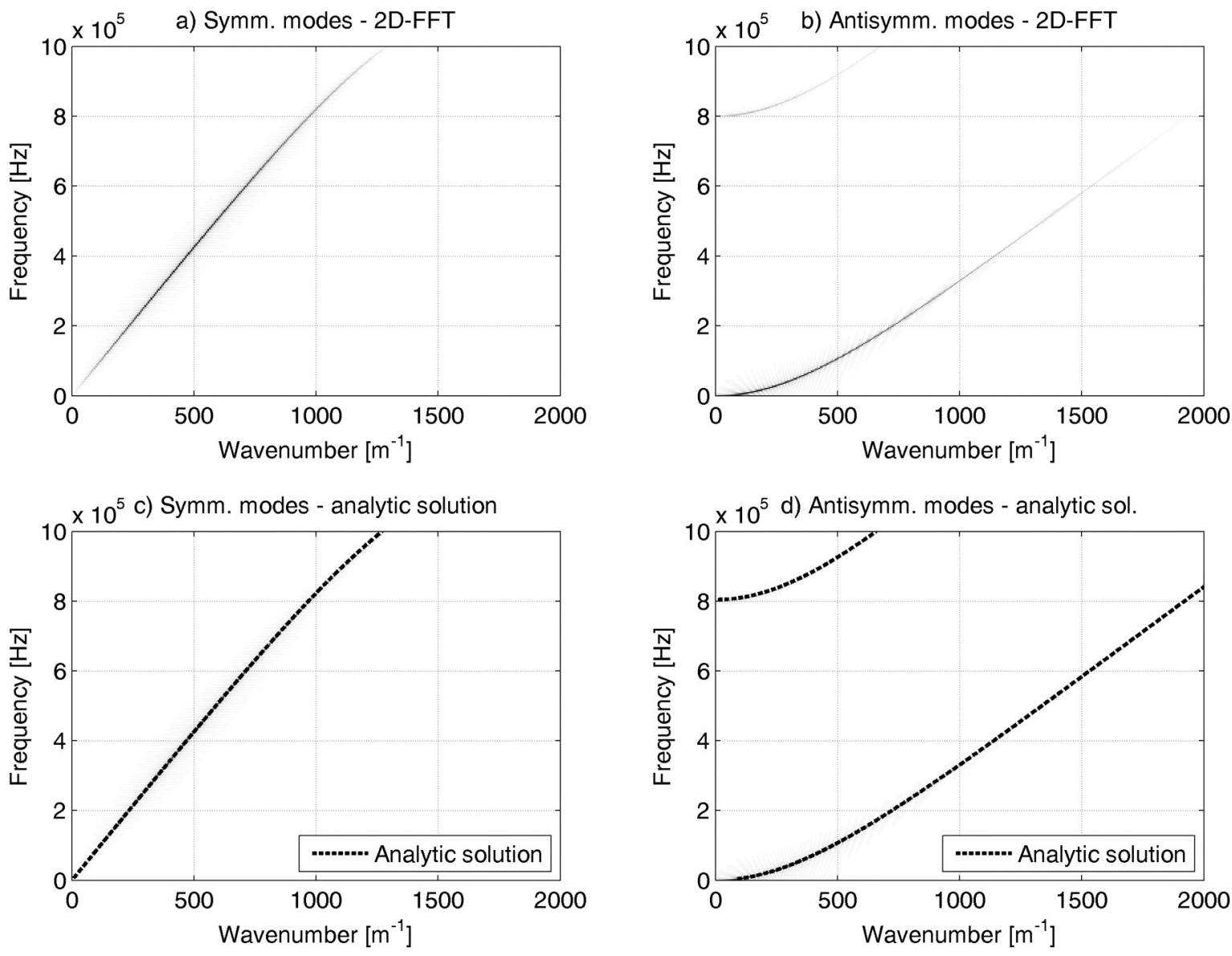

Fig. 4. Result of 2D-FFT and comparison with analytic solution: a) symmetric modes by 2D-FFT, b) antisymmetric modes by 2D-FFT, c) analytic dispersion curves of symmetric modes and d) analytic dispersion curves of antisymmetric modes superimposed on 2D-FFT

\section{Measurement of group velocities}

The measurement of Lamb waves group velocities was performed on a plate with dimensions $800 \times 800 \times 2 \mathrm{~mm}$, which was hanged on a frame by two elastic strings (as can be seen in Fig. 5)

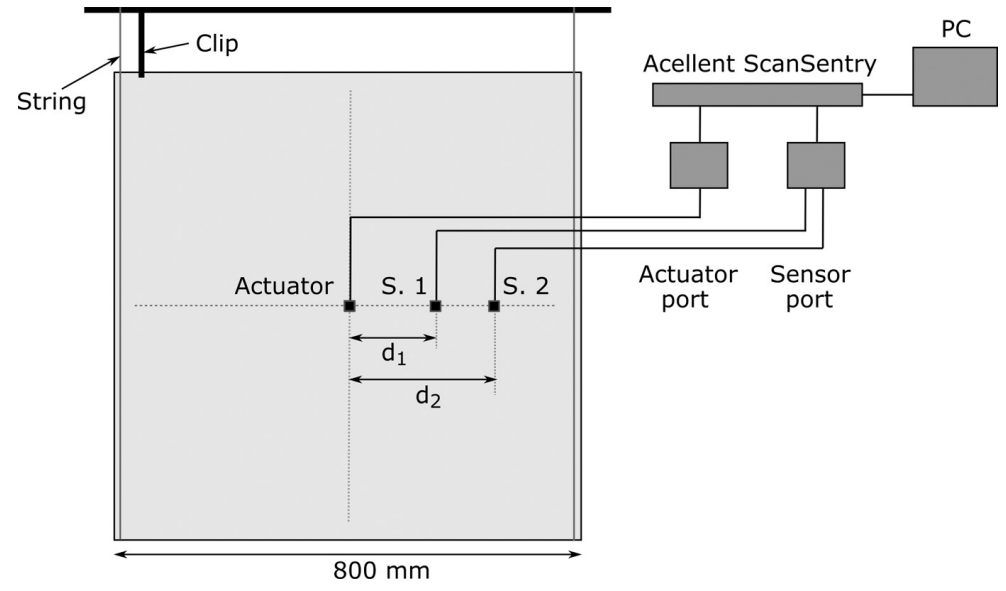

Fig. 5. Measurement set-up: plate hanged on a frame with applied piezoelectric patches 
and secured in vertical position by a clip in the top left corner to minimize contact with the strings. Three transducers were applied on the plate, an actuator and a pair of sensors placed along the investigated direction of propagation. The transducers were piezoelectric patches, specifically DuraAct P-876.SP1. Piezoceramic layer in these patches has dimensions $10 \times 10 \times 0.2 \mathrm{~mm}$ and it is sealed in a protective plastic foil with dimensions $16 \times 13 \times 0.5 \mathrm{~mm}$. More specifications of the transducers can be found in producer's data sheet [16]. The actuator was placed in the centre of the plate. The initial setup of sensors was $d_{1}=150 \mathrm{~mm}$ and $d_{2}=250 \mathrm{~mm}$ measured from the actuator, but it had to be changed to $d_{1}=100 \mathrm{~mm}$ and $d_{2}=200 \mathrm{~mm}$ for some frequencies to avoid overlay of incident and reflected wave packets. Therefore the transducers were bonded to the plate by double-side adhesive tape, which allows their relocation without any damage of the transducers or the plate. On the other hand, this way of attachment can impair quality of recorded signals.

Excitation and recording of signals from piezoelectric transducers was preformed using Acellent ScanSentry, which is specialized device for Lamb wave-based structural health monitoring. It operated with sampling frequency $12 \mathrm{MHz}$ both for actuator and sensors signals. The data acquisition is one-channel, therefore the measurement from the pair of collocated sensors was sequential.

For the measurement of group velocity of certain frequency, it is necessary to isolate this frequency component from recorded signals. It is easier if wave packets with this frequency carry the largest amount of energy. This could be achieved by a choice of an actuation pulse in form of sine burst with this central frequency. It is preferred to reduce influence of geometric dispersion on wave packets, as they are distorted to longer time span and their amplitudes decrease significantly. One possibility is to use actuation pulse limited by window function, e.g. multiplied by Hann window. As presented in [11], higher number of sine periods also reduces the effect of dispersion, on the other hand the pulse lasts a longer period in time and the wave packets may merge together in time signals. Hann-windowed 5-period sine burst (as presented in Fig. 6) was chosen for all measurements. The central frequencies were set in range of 100-500 kHz with step by $50 \mathrm{kHz}$. Measurements were repeated 3-times for each frequency with very low variation of results.

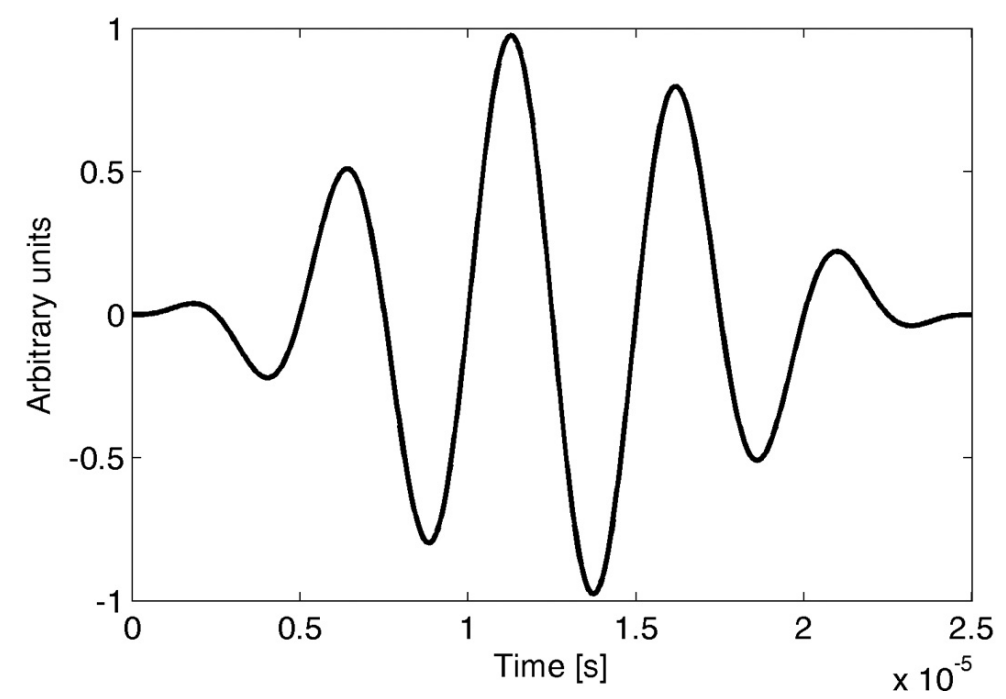

Fig. 6. Actuating pulse: Hann-windowed 5-sine burst, the central frequency in this example is $200 \mathrm{kHz}$ 
a) Measurement - sensor 1

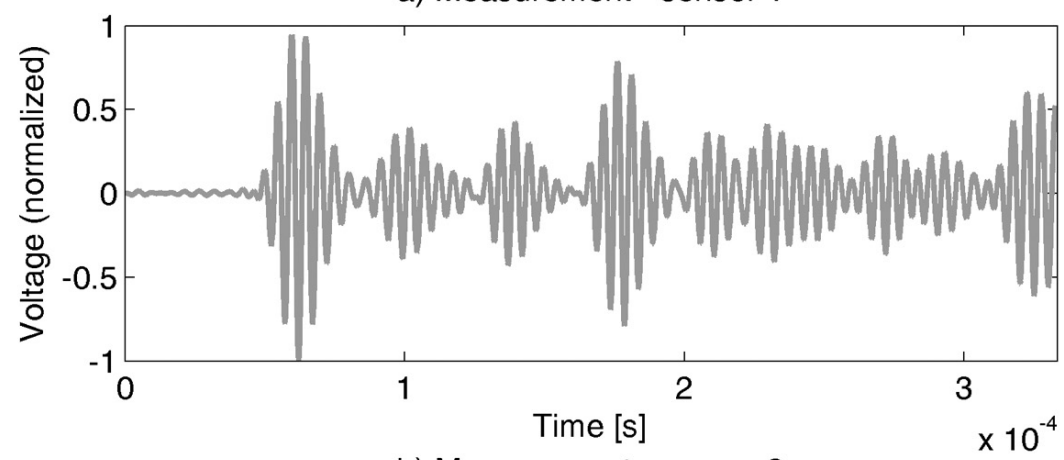

b) Measurement - sensor 2

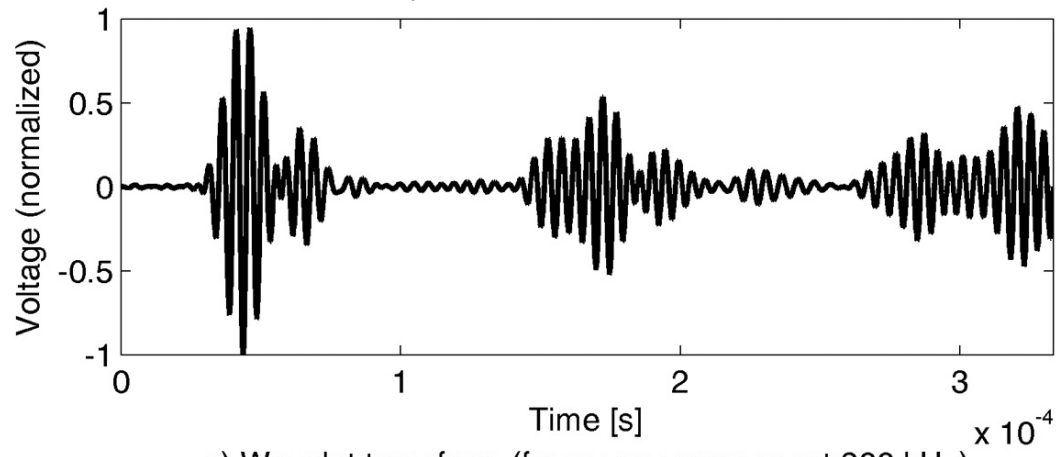

c) Wavelet transform (frequency component $200 \mathrm{kHz}$ )

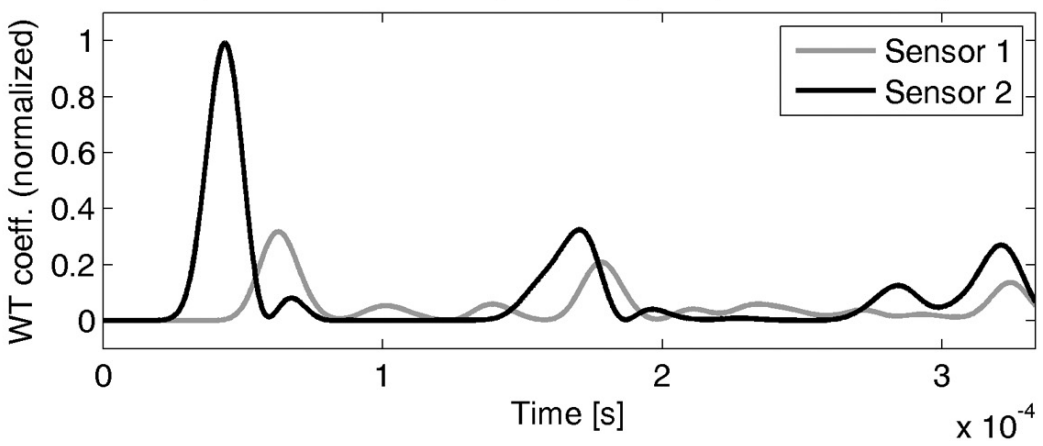

Fig. 7. Measured voltage signals from a pair of sensors and time history of frequency component $f=200 \mathrm{kHz}$

The group velocities were determined from the difference of arrival times detected by the pair of sensors in mutual distance $100 \mathrm{~mm}$. To determine time of arrival of the wave modes, time history of energy of significant frequency has to be separated. The temporal signals from both sensors (Fig. 7a-b) were processed by wavelet transform and the time history of the frequency component (Fig. 7c) was extracted as a corresponding row in the matrix of scalogram (absolute value of the result of wavelet transform). Peaks of wavelet transform coefficient indicate a passage of forward and reflected wave modes.

The propagation of zero-order modes in time and distance from actuator is presented in Fig. 8. The group velocities were calculated from time of arrivals of incident wave packets. It is quite easy to identify the fastest forward $S_{0}$ mode, as it is the first significant peak in the signal, followed by forward $A_{0}$. The dotted lines connecting first two peaks in time signals were prolonged to the edge of plate to identify wave packets reflected from the edge. Especially in lower frequencies, forward mode $A_{0}$ was overlaid by mode $S_{0}$ reflected from the edge and for these measurements the sensors were moved to positions $d_{1}=100 \mathrm{~mm}$ and $d_{2}=200 \mathrm{~mm}$. 


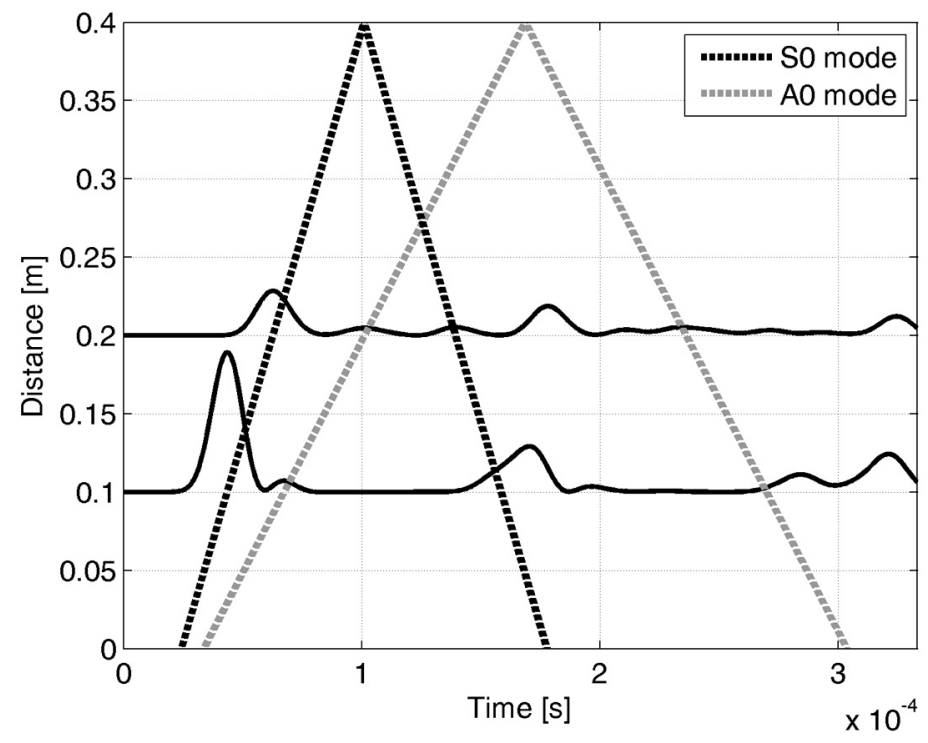

Fig. 8. Propagation of zero-order wave modes through plate in time and travelled distance

The measured group velocities were compared to the results of 2D-FFT and analytic solution, as is shown in Fig. 9. The group velocities from 2D-FFT data were calculated using relation (7) and extrapolated by a polynomial. Results shows good precision in case of mode $A_{0}$ mode and some deviations in case of mode $S_{0}$. These errors are caused mainly by precision in spatial domain, which is given by number of evaluated nodes of the finite element model.

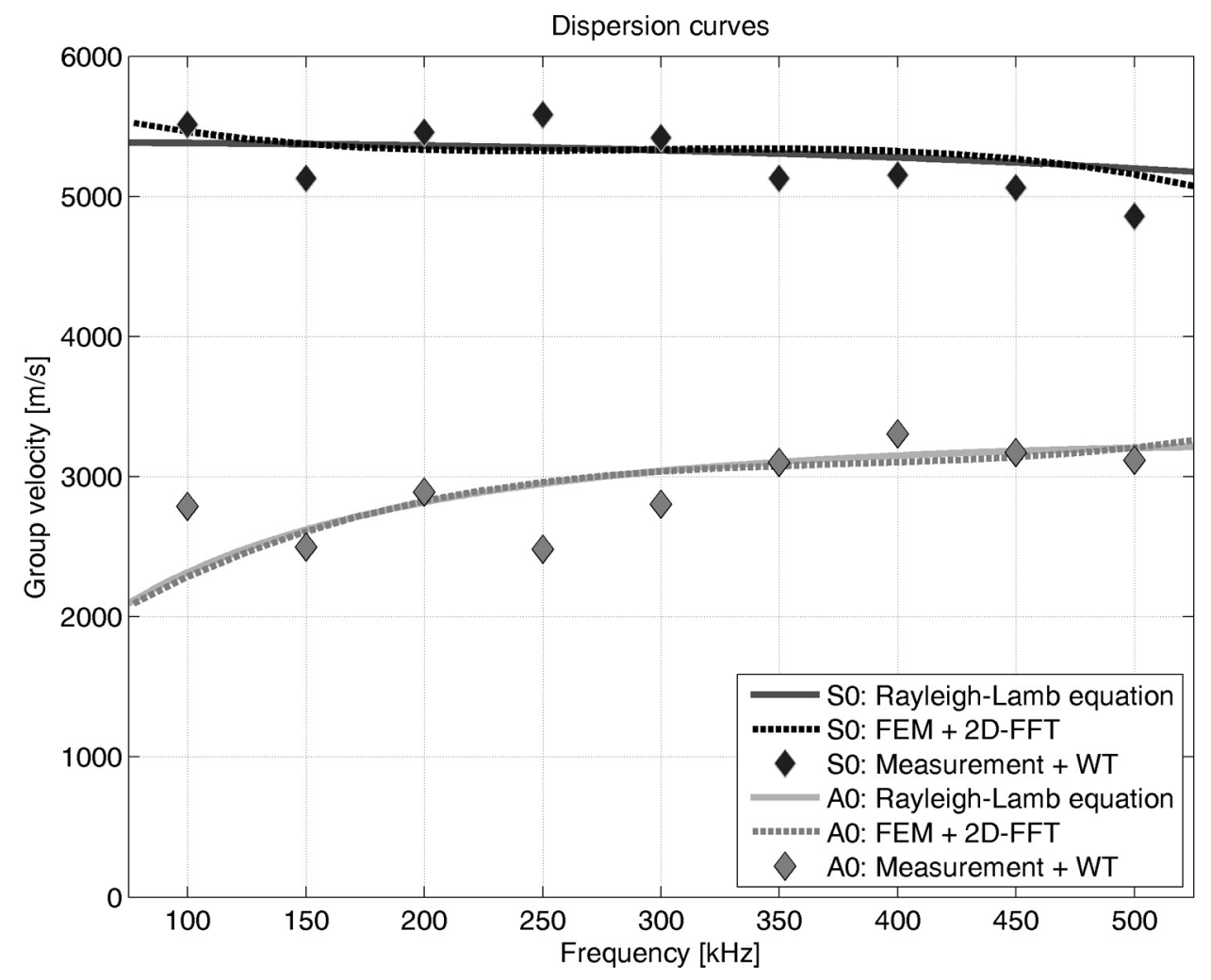

Fig. 9. Dependency of group velocity on frequency of zero-order modes calculated by analytic solution, FEM + 2D-FFT and from measured data + wavelet transform 
Experimental results oscillate around theoretical dispersion curve with average difference $3.4 \%$ in case of $S_{0}$ and $6.4 \%$ for mode $A_{0}$. The reason for this is that the mode $S_{0}$ is not very dispersive in this frequency range and it can be easily found in time signals. On the other side $A_{0}$ is more dispersive in low frequency range, which is the likely source of errors. The influence of dispersion decreases with increasing frequency.

\section{Conclusion}

Dispersion curves (group velocity dependent on frequency) of two zero-order Lamb wave modes in aluminum plate were obtained by two methods: two-dimensional Fourier transform applied on data from the finite element analysis and measurement using piezoelectric transducers.

2D-FFT provides well approximated dispersion curves in $k$ - $f$ domain, which correlate with dispersion curves calculated by solution of Rayleigh-Lamb equation. The precision of calculation of group velocity depends on number of "sensors" and time increment of input data, i.e. samples for Fast Fourier Transform. In this case sufficient precision was achieved using 4096 samples.

Measurement of group velocities by piezoelectric transducers and signal processing by wavelet transform proves to be more accurate in frequencies with lower effect of geometric dispersion. In case of symmetric mode $S_{0}$ it oscillates around analytic curve with average difference $3.4 \%$ and $6.4 \%$ in case of anti-symmetric mode $A_{0}$.

Both proposed methods showed sufficient precision for identification of zero-order Lamb wave modes in time signals by determination of their group velocity. Their main advantage is applicability on a wider set of structures in comparison with analytical solution.

\section{Acknowledgements}

This publication was supported by the project LO1506 of the Czech Ministry of Education, Youth and Sports.

\section{References}

[1] Alleyne, D. N., Cawley, P., A two-dimensional Fourier transform method for the measurement of propagating multimode signals, Journal of the Acoustical Society of America 89 (3) (1991) 1159-1168. https://doi.org/10.1121/1.400530

[2] Ambrozinski, L., Packo, P., Stepinski, T., Uhl, T., Ultrasonic guided waves based method for SHM-simulations and an experimental test, Proceedings of the 5th World Conference on Structural Control and Monitoring, Tokyo, 2010.

[3] Dalton, R. P., Cawley, P., Lowe, M. J. S., The potential of guided waves for monitoring large areas of metallic aircraft fuselage structure, Journal of Nondestructive Evaluation 20 (1) (2001) 29-46. https://doi.org/10.1023/A:1010601829968

[4] Gao, W., Glorieux, C., Thoen, J., Laser ultrasonic study of Lamb waves: Determination of the thickness and velocities of a thin plate, International Journal of Engineering Science 41 (2) (2003) 219-228. https://doi.org/10.1016/S0020-7225(02)00150-7

[5] Graff, K. F., Wave motion in elastic solids, Courier Corporation, 2012.

[6] Hayashi, T., Kawashima, K., Single mode extraction from multiple modes of Lamb wave and its application to defect detection, JSME International Journal Series A Solid Mechanics and Material Engineering 46 (4) (2003) 620-626. https://doi.org/10.1299/jsmea.46.620

[7] Hora, P., Thick plate toolbox I., Research Report Z13xx/02, Institute of Thermomechanics of the CAS, Laboratory of Material Diagnostics in Pilsen, Pilsen, 2002. (in Czech) 
[8] Hora, P., Červená, O., Determination of Lamb wave dispersion curves by means of Fourier transform, Applied and Computational Mechanics 6 (1) (2012) 5-16.

[9] Hu, N., Shimomukai, T., Fukunaga, H., Su, Z., Damage identification of metallic structures using $\mathrm{A}_{0}$ mode of Lamb waves, Structural Health Monitoring 7(3) (2008) 271-285. https://doi.org/10.1177/1475921708090566

[10] Imano, K., Experimental study on the mode conversion of Lamb wave using a metal plate having a notch type defect, International Journal of the Society of Materials Engineering for Resources 19 (1-2) (2013) 20-23. https://doi.org/10.5188/ijsmer.19.20

[11] Ip, K. H., Tse, P. W., Tam, H. Y., Extraction of patch-induced Lamb waves using a wavelet transform, Smart Materials and Structures 13 (4) (2004) 861-872. https://doi.org/10.1088/0964-1726/13/4/024

[12] Jeong, H., Jang, Y.S., Fracture source location in thin plates using the wavelet transform of dispersive waves, IEEE Transactions on Ultrasonics, Ferroelectrics, and Frequency Control 47(3) (2000) 612-619. https://doi.org/10.1109/58.842048

[13] Liu, X., Zhou, C., Jiang, Z., Damage localization in plate-like structure using built-in PZT sensor network, Smart Structures and Systems 9 (1) (2012) 21-33.

https://doi.org/10.12989/sss.2012.9.1.021

[14] Prosser, W.H., Seale, M.D., Smith, B.T., Time-frequency analysis of the dispersion of Lamb modes, The Journal of the Acoustical Society of America 105 (5) (1999) 2669-2676. https://doi.org/10.1121/1.426883

[15] Ostachowicz, W., Kudela, P., Malinowski, P., Wandowski, T., Damage localisation in plate-like structures based on PZT sensors, Mechanical Systems and Signal Processing 23 (6) (2009) 1805-1829. https://doi.org/10.1016/j.ymssp.2008.10.011

[16] PI Datasheet P-876, retrieved May 15, 2017, from https://www.physikinstrumente.com. 